\title{
Psicooncología
}

ISSN: $1696-7240$

\section{Distrés psicológico, miedo a la recurrencia del cáncer y calidad de vida relacionada con la salud en sobrevivientes de cáncer de mama venezolanas}

\author{
José Javier Altuve Burgos ${ }^{1, *}$
}

Recibido: 22 de julio de 2020 / Aceptado: 31 de agosto de 2020

Resumen: Objetivo: El objetivo de la investigación fue determinar las relaciones entre el distrés psicológico, el miedo a la recurrencia del Cáncer (MRC) y la calidad de vida relacionada con la salud (CDVRS) en pacientes sobrevivientes de cáncer de mama venezolanas. Método: El estudio fue de corte correlacional y participaron 203 pacientes sobrevivientes de Cáncer de mama, de edades entre 24 y 75 años. Se emplearon las escalas de Ansiedad y Depresión Hospitalaria (HADS), de preocupaciones relacionadas con el Cáncer y de salud global SF - 12 versión 2. Resultados: Se reportaron niveles bajos de distrés psicológico, aunque con predominio de niveles medios de ansiedad y de MRC. La prevalencia de ansiedad elevada fue de $23 \%$, de depresión elevada de $7 \%$, de distrés psicológico de $18 \%$ y de MRC de $36 \%$. El distres psicológico y MRC correlacionaron de forma positiva y significativa, aunque con fuerza de baja a moderada. La CDVRS estuvo en un rango normal y correlacionó de forma inversa y significativa con el distrés psicológico así como también con el MRC. Conclusión: El distrés psicológico se encuentra asociado, aunque no en gran magnitud, con el MRC y que ambos se asocian con un deterioro de la CDVRS en las pacientes sobrevivientes de Cáncer de mama venezolanas. Además, el MRC y la CDVRS correlacionaron de forma positiva, baja y significativa con el tiempo de supervivencia de las pacientes.

Palabras clave: Cáncer de mama, distrés psicológico, miedo a la recurrencia del cáncer, calidad de vida relacionada con la salud, psicooncología.

\section{[en] Psychological distress, fear of cancer recurrence and health-related quality of life in Venezuelan surviving with breast Cancer}

\footnotetext{
Abstract: Objetive: The objetivo of the following study was to determine the relationships between psychological distress, fear of cancer recurrence (FCR) and health - related quality of life (HQoL) in Venezuelan breast cancer survivors. The study was with a correlational cut, 203 breast cancer survivors between the age of 24 and 75 participated. Method: The scales of Hospital Anxiety and Depression (HADS), Cancer- related Worry and Global Health SF - 12 version 2 were used. Results: Low levels of psychological distress were reported, but with a predominance of means levels of anxiety and FCR. The prevalence of high anxiety was $23 \%$, depression $7 \%$ an FCR $36 \%$. Psychological distrees and FCR correlated positively and significantly, although with low to moderate. HQoL was in a normal range and correlated inversely and significantly with psychological distress, as well as with FCR. Conclusion: The psychological distress and MRC are associated, but although not in large magnitude, and that both

1 José Javier Altuve Burgos, Facultad de Humanidades y Educación, Escuela de Psicología, Universidad Central de Venezuela, Caracas, Venezuela.

E-mail: a.altuve.07@gmail.com

* Dirección de Correspondencia: Servicio de Psicología, Servicio Oncológico Hospitalario del Instituto Venezolano de los Seguros Sociales, Caracas, Venezuela. E-mail: a.altuve.07@gmail.com
} 
are associated with a deterioration of HQoL in Venezuelan breast cancer survivors. FCR nd HQoL correlated positively and significantly with time survival of patients.

Keywords: Breast cancer, psychological distress, fear of cancer recurrence, health - related quality of life, psychooncology.

Sumario: 1. Introducción 2. Método 3. Análisis estadístico 4. Resultados 5. Discusión 6. Conclusiones 7. Referencias bibliográficas

Cómo citar: Altuve Burgos JJ. Distres psicológico, miedo a la recurrencia del cáncer y calidad de vida relacionada con la salud en sobrevivientes de cáncer de mama venezolanas. Psicooncología 2020; 17: 239-253. doi:10.5209/psic.71053

\section{Introducción}

Las enfermedades crónicas representan un desafío para la salud de los individuos y sus familias, puesto que menoscaban una parte de su calidad de vida global. En el contexto de las enfermedades crónicas se ha empleado frecuentemente el término calidad de vida relacionada con la salud (CDVRS) ${ }^{(1)}$, que es un fenómeno subjetivo que se refiere a la valoración, tanto positiva como negativa, de una persona sobre su enfermedad y sus tratamientos, así como también del impacto que tiene para su bienestar en las dimensiones físicas, cognitivas, emocionales y sociales ${ }^{(2)}$.

El cáncer es una de las enfermedades crónicas que más temor suscita en las personas, dado que se asocia directamente con el sufrimiento, el dolor y la muerte en casi todas las culturas ${ }^{(3)}$. Específicamente, el cáncer de mama es una de las patologías que se diagnostica frecuentemente en las mujeres y representa el $16 \%$ de todos los canceres femeninos ${ }^{(4)}$. En Venezuela, siguiendo las últimas cifras de mortalidad, se reportó que alrededor de 2.067 mujeres fallecieron por cáncer de mama, pero a pesar de está cifra, su tasa de supervivencia es superior a los dos años ${ }^{(5)}$.

Es frecuente que las pacientes con cáncer de mama experimenten un fuerte impacto emocional ante el diagnóstico ${ }^{(6)}$. A este impacto emocional se le ha conocido como distrés psicológico y es una experiencia emocional desagradable, multifactorial, de naturaleza psicológica (cognitiva, conductual y emocional), social y/o espiritual que tiende a incidir en la habilidad para afrontar efectivamente el cáncer, sus síntomas y tratamientos ${ }^{(7,8)}$. El distrés es común en toda la trayectoria y fases de la enfermedad ${ }^{(8)}$. Alrededor del $20 \%$ al $47 \%$ de los pacientes oncológicos informan niveles significativos de distrés psicológico, que no son sugestivos de un trastorno adaptativo o del ánimo ${ }^{(9)}$. El distrés psicológico puede varia de acuerdo al tipo de cáncer, con una prevalencia general de $35,1 \%$, específicamente, en pacientes con cáncer de mama, se ha reportado una prevalencia de $32,8 \%{ }^{(10)}$.

El distrés elevado se ha asociado con una disminución de la CDVRS de los pacientes con cáncer de mama ${ }^{(6,8)}$. Este deterioro afecta el funcionamiento físico, el desempeño de los roles en la vida diaria, promueve a menor adherencia a los tratamientos, poca satisfacción con la atención médica y, posiblemente, una menor supervivencia ${ }^{(1,6,11,12)}$. Aunque el distrés puede ser fluctuante a lo largo de la enfermedad ${ }^{(9,10)}$, las pacientes que sobreviven de cáncer de mama tienden a tener un mejor ajuste psicológico luego de los tratamientos, pero al mismo tiempo, pueden reportar niveles moderados de distrés asociados a preocupaciones relativas a la enfermedad ${ }^{(13)}$. 
Una de las preocupaciones más frecuentes en los sobrevivientes de cáncer es sobre la posibilidad de que su enfermedad se vuelva a desarrollar a futuro ${ }^{(14,15)}$. Así, el miedo a la recurrencia del cáncer (MRC) ha sido un aspecto muy común en los pacientes con cáncer de mama ${ }^{(16-18)}$. Se ha definido frecuentemente como el miedo a que el cáncer pueda volver o progresar en el mismo lugar o en otra parte del cuerpo, o bien, al grado de preocupación que reportan los pacientes acerca de cambios que podrían indicar que el cáncer va progresar a futuro ${ }^{(15,17,19)}$. Este miedo es una respuesta reactiva adecuada y esperada, de la que los pacientes son conscientes y puede ser adaptativa y funcional en un primer momento, pero en niveles disfuncionales pueden afectar el bienestar y la calidad de vida ${ }^{(14-20)}$. La prevalencia del MRC puede variar de acuerdo al sitio del cáncer. Alrededor del $49 \%$ de los casos han reportado niveles moderados de MRC, mientras que solo $7 \%$ niveles altos ${ }^{(19,20)}$. Específicamente, en las sobrevivientes de cáncer de mama se ha reportado hasta un $70 \%{ }^{(11,21)}$. Otra revisión ${ }^{(22)}$ reportó niveles bajos en un $82 \%$ y niveles altos en un $6 \%$. Este miedo suele ser estable en el tiempo, pero tiende a decrecer en los meses posteriores al diagnóstico, aunque no en todos los casos es así ${ }^{177,20)}$.

Se ha reportado evidencia de la relación, frecuentemente moderadas, entre el distres y el MRC ${ }^{(17,23-26)}$. Mientras que otros estudios ${ }^{(27,28)}$ no encontrado relación entre estas variables, o bien, que pueden manifestarse en conjunto. El MRC al ser elevado afecta la CDVRS, deteriorando la salud física y mental de los pacientes oncológicos, que provoca en ellos impedimentos importantes en el desenvolvimiento en la vida diaria $^{(17-26,29-31)}$. Se ha informado que las pacientes con cáncer de mama que informan una mejor CDVRS, tienden a tener menor MRC a lo largo de la enfermedad ${ }^{(22,32)}$.

En tal sentido, la relación entre el distrés psicológico, el MRC y la CDVRS en los pacientes oncológicos ha sido ampliamente reportada en estudios realizados en diversos países, pero en Venezuela, siguiendo la revisión bibliográfica, no se han reportado investigaciones al respecto. Considerando que el cáncer de mama es uno de los canceres que frecuentemente se diagnostica en el país y que cuenta con una mayor tasa de supervivencia, el siguiente estudio, de tipo correlacional ${ }^{(33)}$, tuvo como objetivo determinar las relaciones entre el distrés psicológico, el miedo a la recurrencia del cáncer y la calidad de vida relacionada con la salud en una muestra de pacientes con cáncer de mama venezolanas.

\section{Método}

\section{Participantes}

Se contó con la participación de 203 mujeres diagnosticadas con cáncer de mama, de edades entre 24 y 75 años. El muestreo realizado en la investigación fue no probabilístico $^{(33)}$. Los criterios que se siguieron para la elección de la muestra fueron que todas las pacientes estuvieran en la fase de supervivencia ( $\geq 2$ años), mayores a los 18 años de edad, que reportaran en la consulta su participación voluntaria, expresaran sentirse bien de ánimo, que no reportaran síntomas físicos, tales como dolor, fatiga e insomnio, además de dificultades cognitivas relacionadas con la atención, concentración y comprensión, que supieran leer y escribir. En la tabla 1 se proporciona la información sociodemográfica y de salud de la muestra de pacientes. 
Tabla 1. Perfil sociodemográfico y de salud de la muestra.

\begin{tabular}{lcc}
\hline Edad & $\mathrm{N}(\%)$ & $\mathrm{M}(\mathrm{DT})-$ IC $95 \%$ \\
$\leq 39$ & $14(7 \%)$ & \\
$40-55$ & $87(43 \%)$ & 56,$8 ;(12,1)-55-58$ \\
$\geq 56$ & $102(50 \%)$ & \\
\hline Estrato socioeconómico & $2(1 \%)$ \\
$\quad$ Alto & $93(46 \%)$ \\
$\quad$ Medio & $108(53 \%)$ \\
$\quad$ Bajo & \\
\hline Estado civil & $66(33 \%)$ \\
$\quad$ Casadas & $98(48 \%)$ \\
Solteras & $13(6 \%)$ \\
Divorciadas & $26(13 \%)$ \\
$\quad$ Viudas & \\
\hline Nivel de instrucción & $51(25 \%)$ \\
Primaria completa & $105(52 \%)$ \\
Secundaria completa & $47(23 \%)$ \\
Universitaria & \\
\hline Madres &
\end{tabular}

\section{Madres}

$\mathrm{Si}$

$167(82 \%)$

No

$36(18 \%)$

\section{Actividad Laboral}

$\mathrm{Si}$

$156(77 \%)$

No

$47(23 \%)$

\section{Comorbilidad otras enfermedades}

$\mathrm{Si}$

$58(29 \%)$

No

$145(71 \%)$

\section{Antecedentes psiquiátricos}

$\mathrm{Si}$

$34(16 \%)$

No

$169(83 \%)$

\section{Tiempo con el diagnostico}

$<4$ años

$\geq 5$ años

$164(80 \%)$

$39(20 \%)$

3,$48 ;(3,37)-3,02-3,95$

\section{Estadio Inicial}

In situ (Estadio 0)

Estadio I 
Estadio II

Estadio III

Estadio IV
$74(37 \%)$

$85(42 \%)$

$15(7 \%)$

\section{Tratamiento recibido}

Quimioterapia

$143(71 \%)$

Radioterapia

$27(13 \%)$

$\mathrm{QT}+\mathrm{RT}$

$33(16 \%)$

\section{Cirugía por Mastectomía}

Radical

$149(73 \%)$

Parcial

$54(27 \%)$

\section{Cirugía Reconstructiva}

\begin{tabular}{lc}
$\mathrm{Si}$ & $134(66 \%)$ \\
\hline No & $69(34 \%)$
\end{tabular}

Tiempo de Supervivencia (luego de la

fase de tratamiento)

\begin{tabular}{|c|c|c|}
\hline 2 - 5 años & $174(86 \%)$ & $3,01(2,86) / 2,61-3,41$ \\
\hline$>6$ años & $29(14 \%)$ & \\
\hline
\end{tabular}

\section{Complicaciones físicas (últimos 3 meses)}

$\mathrm{Si}$

$40(20 \%)$

No

$163(80 \%)$

\section{Manejo de los síntomas de la enfermedad}

Bueno

Regular

Mal
$159(78 \%)$

$34(17 \%)$

$10(5 \%)$

\section{Procedimiento}

A las pacientes sobrevivientes de cáncer de mama que asistían a la consulta de seguimiento médico de la enfermedad en el servicio de oncología médica, del Servicio Oncológico Hospitalario de Instituto Venezolano de los Seguros Sociales, ubicado en la Ciudad de Caracas, Venezuela, se les pidió su participación en el estudio. Luego de que se verificara si las pacientes cumplían con los criterios de inclusión de la muestra y aceptaran participar voluntariamente, se les explicó los fines de la investigación y se les hizo entrega del consentimiento informado, en el que quedaban expuestos todos los lineamientos éticos de la investigación. Para la aplicación de las medidas de auto-informe empleadas, se contó con la colaboración del personal de oncología médica y de psicología del centro hospitalario, quienes las aplicaron a las pacientes las escalas durante el desarrollo de las respectivas consultas. La aplicación se llevo a cabo desde el mes de junio de 2019 hasta el mes de febrero del 2020. 


\section{Instrumentos}

Datos sociodemográficos y de salud. Se empleó una escala creada por el autor en la que se recogen datos sobre la edad, el estrato socioeconómico, estado civil, el grado de instrucción, la actividad laboral, etc. Además de datos relacionados con la salud tales como la existencia de comorbilidades por otras afecciones, antecedentes psiquiátricos, los años con el diagnóstico, dificultades físicas actuales y manejo de los síntomas de la enfermedad. Además, la información sobre el estadio inicial de la enfermedad, el tipo de tratamiento recibido, la cirugía realizada y el tiempo especifico de sobrevida, luego de la aplicación de los tratamientos e intervenciones quirúrgicas, fueron consultados en las historias clínicas de cada paciente.

Escala de Ansiedad y Depresión Hospitalaria (HADS) de Zigmond y Snait ${ }^{(34)}$. Se empleó la versión adaptada de Rico et al.(35). La escala evalúa la ansiedad y depresión en el contexto hospitalario. Esta conformada por 14 reactivos, cada sub escala, de ansiedad y depresión, están conformadas por 7 ítems cada una, que se mide en una escala de 0 a 3 puntos. La suma de ambas sub escalas conforman la puntuación global de distrés psicológico (rango de puntaje: 0-42). Puntajes mayores en las respectivas escalas indican mayor presencia de síntomas ansiosos y depresivos o bien de distrés psicológico en el puntaje total. Los índices de confiabilidad de esta versión fueron de $\alpha_{\text {ansiedad }}=0,779 \alpha_{\text {depresión }}=0,776 \alpha_{\text {distrés }}=0,855$ (CITA). Los índices de confiabilidad obtenidos en la muestra de este estudio fueron: $\alpha_{\text {ansiedad }}=0,794, \alpha_{\text {depresión }}=0,752 \alpha_{\text {distrés }}=0,863$.

Escala de preocupaciones relacionadas con el cáncer. Se empleo la escala de preocupaciones relacionadas con el cáncer diseñada por Deimiling, et al. ${ }^{(36)}$, basada en el trabajo realizado por Gotay y Murakoka ${ }^{(37)}$. Esta escala evalúa MRC y esta conformada por 4 ítems, que mediante un estudio realizado para fines de esta investigación, fueron traducidos y adaptados a la población venezolana siguiendo las directrices propuestas por Muniz, et al ${ }^{(38)}$. Luego de realizarse estas fases, resultó la siguiente versión en castellano definitiva: 1 . "A veces me preocupa que los síntomas que experimento puedan indicar recurrencia del cáncer"; 2. "Me preocupan los resultados de mis exámenes diagnósticos a futuro"; 3. "Me inquieta que mi cáncer vuelva"; 4. "Me preocupa que otro tipo de cáncer pueda aparecer". Cada reactivo se responde en una escala continua de 5 puntos, desde: " 1 = Fuertemente de acuerdo" a " 5 = Fuertemente en desacuerdo". Altos puntaje indican mayor MRC. El índice de confiabilidad reportado de la escala fue: $\alpha$ Miedo a la recurrencia $=0,84^{(36)}$. El estudio piloto realizado por el autor para fines de esta investigación, que contó con 100 pacientes con cáncer de mama, de edades entre 25 a 70 años, que se encontraban en la fase de supervivencia de la enfermedad, reportó una consistencia interna $\alpha_{\text {Miedo a la recurrencia }}=0,79$, resultando aceptable.

Cuestionario breve de salud general SF -12 - versión 2. Escala original de Ware et al. ${ }^{(39)}$. Se empleó versión adaptada y validada en Venezuela por Hernández, et al. ${ }^{(40)}$. Es una escala que evalúa la calidad de vida relacionada con la salud que consta 12 ítems integrados en ocho dimensiones: función física (dos), rol físico (dos), dolor corporal (uno), salud general (uno), vitalidad (uno), función social (uno), rol emocional (dos) y salud mental (dos). Estas dimensiones se agrupan a su vez en dos componentes sumatorios: salud física (rango de puntaje: 6 - 20) y salud mental (rango de puntaje: 6 - 27), que dan un puntaje total de CDVRS (rango de puntaje: 12 - 47). Puntajes mayores indican mejor CDVRS. El número 
de opciones de respuesta entre los ítems oscila entre tres y seis puntos. Puntajes mayores indican una alta salud física, mental o de CDVRS en su puntaje total. Los índices de confiabilidad obtenidos en el estudio de Hernández et al. ${ }^{(40)}$ fue. $\alpha$ salud fisica $=0,75, \alpha_{\text {Salud mental }}=0,78, \alpha_{\text {CDVRS }}=0,83$. Para la muestra de pacientes con cáncer de mama sobrevivientes de este estudio se reportaron los siguientes índices: $\alpha_{\text {salud fisica }}$ $=0,74, \alpha_{\text {Salud mental }}=0,77$ y $\alpha_{\text {CDVRS }}=0,88$.

\section{Análisis estadístico}

Los datos fueron computados en el programa estadístico SPSS versión 24. Para la realización de los análisis correspondientes, se calcularon los estadísticos descriptivos y de frecuencia para reportar el perfil sociodemográfico y de salud así como la prevalencia de distrés psicológico y miedo a la recurrencia en la muestra. Se empleó la prueba Momento de Pearson y de Rho de Spearman para determinar las correlaciones entre las variables de estudio.

\section{Resultados}

En la tabla 2 se pueden observar los estadísticos descriptivos de la muestra de pacientes de cáncer de mama sobrevivientes obtenidas en las variables de estudio. Se puede observar que las pacientes con cáncer de mama reportan, en promedio, niveles bajos de depresión, pero niveles medios de ansiedad. En suma, el distrés psicológico resultó encontrarse, en promedio, en niveles bajos. El miedo a la recurrencia por su parte reportó una tendencia a los niveles moderados. Finalmente, en cuando a los indicadores de salud se observa que las pacientes tienden a tener puntajes elevados en su salud mental y física y en los puntajes de salud global de CDVRS, encontrándose dentro de los rangos normales.

Tabla 2. Estadísticos descriptivos de las variables de estudio.

\begin{tabular}{ccc}
\hline & N (\%) & M; (DT)*/ IC 95\% \\
\hline $\begin{array}{c}\text { Distres Psicológico } \\
\text { Ansiedad }\end{array}$ & & \\
Bajo & $77(38 \%)$ & \\
Medio & $80(39 \%)$ & 5,$86 ;(3,56) / 5,37-6,36$ \\
Alto & $\mathbf{4 6}(\mathbf{2 3 \%})$ & \\
\hline Depresión & & \\
Baja & $154(76 \%)$ & \\
Medio & $34(17 \%)$ & 3,$82 ;(3,23) / 3,37-4,27$ \\
Alto & $\mathbf{1 5 ( 7 \% )}$ & \\
\hline Distres total & $109(54 \%)$ & \\
Bajo & $57(28 \%)$ & 9,$68 ;(5,78) / 8.8-10,4$ \\
Medio & $\mathbf{3 7 ( 1 8 \% )}$ & \\
Alto & & \\
\hline
\end{tabular}




\begin{tabular}{|c|c|c|}
\hline \multicolumn{3}{|l|}{ Miedo a la Recurrencia } \\
\hline Bajo & $23(11 \%)$ & \\
\hline Medio & $108(53 \%)$ & 12,$53 ;(5,04) / 11,8-13,2$ \\
\hline Alto & $72(36 \%)$ & \\
\hline \multicolumn{3}{|c|}{ Calidad de vida relacionada con la salud. } \\
\hline Salud Física & & 18,$4 ;(3,21) / 15,3-18,9$ \\
\hline Salud Mental & & 15,$3 ;(4,25) / 13,2-16,2$ \\
\hline Salud Global (CDVRS) & & 32,$3 ;(4,20) / 31,4-34,7$ \\
\hline
\end{tabular}

Finalmente, en la tabla 3 se aprecian la correlaciones entre las variables de estudio. La ansiedad $(r=0,423 ; p=<0,01)$ y la depresión $(r=0,217 ; p=<0,01)$ correlacionan positiva y significativa con el MRC, e igualmente con el total de distrés psicológico ( $\mathrm{r}$ $=0,281 ; \mathrm{p}=<0,01)$ también se aprecia esta relación. Tal como se puede notar, estas correlaciones se reportan de magnitud baja y moderada, por lo que su interpretación ha de considerarse con precaución.

La ansiedad correlaciona inversa y significativamente con los indicadores de salud física $(r=-0,465 \mathrm{p}=<0,01)$ y mental $(\mathrm{r}=-0,663 \mathrm{p}=<0,05)$, al igual que con el total de CDVRS $(r=-0,624<0,05)$. La depresión obtiene correlaciones inversas y significativas, pero moderadas, con la salud física $(r=-0,521 \mathrm{p}=<0,01)$, y la CDVRS total $(\mathrm{r}=-0,513 ; \mathrm{p}$ $=<0,01)$, a excepción de la salud mental, en la que reporta una mayor fuerza $(\mathrm{r}=-0,645$; $\mathrm{p}=<0,01)$. El distrés psicológico también se asoció de forma inversa y significativa con la salud física $(\mathrm{r}=-0,483 ; \mathrm{p}<0,01)$, la salud mental $(\mathrm{r}=-0,649 ; \mathrm{p}=<0,01)$ y el total de CDVRS $(r=-0,630 ; p=<0,01)$.

Asimismo, el MRC correlacionó de forma inversa y significativa, pero con magnitudes moderadas, tanto con la salud fisca $(r=-0,242 ; p=<0,01)$, la salud mental $(r=-0,346 ; p$ $=<0,01)$ y el puntaje total de CDVRS $(r=-0,435 ; \mathrm{p}=<0,01)$. Esto indica que a mayor presencia de distrés psicológico, mayor MRC y, a mayor presencia de ambos, menor CDVRS en las pacientes sobrevivientes de cáncer de mama.

Tabla 3. Correlaciones entre las variables de estudio.

\begin{tabular}{|c|c|c|c|c|c|c|}
\hline & 2 & 3 & 4 & 5 & 6 & 7 \\
\hline 1. Ansiedad &, $447 * *$ &, $459 * *$ &, $423 * *$ &,$- 465 * *$ &,$- 663 *$ &,$- 624 *$ \\
\hline 2. Depresión & &, $835 * *$ &, $217 * *$ &,$- 521 * *$ &,$- 645^{*}$ &,$- 513 *$ \\
\hline 3. Distrés psicológico & & &, $281 * *$ &,$- 483 * *$ &,$- 649 *$ &,$- 630^{*}$ \\
\hline 4. Miedo a la recurrencia & & & &,$- 242 * *$ &,$- 346^{* *}$ &,$- 435 * *$ \\
\hline 5. Salud Física & & & & &, $677^{*}$ &, $598 *$ \\
\hline 6. Salud Mental & & & & & & ,619* \\
\hline \multicolumn{7}{|l|}{ 7. CDVRS } \\
\hline $\mathrm{N}=203$ & & & & & & \\
\hline $\begin{array}{l}\text { Prueba Momento de Pear } \\
\text { * La relación es significat }\end{array}$ & $0,05 /$ & 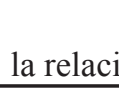 & . & & & \\
\hline
\end{tabular}


Se exploraron las correlaciones entre las variables de estudio con la edad, el estadio inicial y tiempo de supervivencia, considerado desde la finalización de los tratamientos e intervenciones de la enfermedad. Tal como se puede apreciar en la tabla 4, la ansiedad, la depresión y el distrés psicológico total no correlacionaron con estas variables. En cambio, el MRC correlaciono de forma baja y positiva, pero significativa $(r=0,157: \mathrm{p}$ $<0,05)$ con el tiempo de supervivencia, indicando que a mayor tiempo de sobrevida, más manifestación de $\mathrm{MRC}$, en tanto que con las demás variables no hubo relación. La misma dirección y magnitud se aprecia con la CDVRS en la relación con el tiempo de superviviencia $(r=0,163: \mathrm{p}<0,05)$, siendo significativa e indicando que un mayor tiempo de superviviencia se asocia a mayor percepción de CDVRS. Asimismo, la CDVRS no correlacionó de forma significativa con la edad ni el estadio inicial de la enfermedad de las pacientes.

Tabla 4. Correlaciones entre las variables de estudio, la edad, el estadio inicial y el tiempo de supervivencia.

\begin{tabular}{lccc} 
& Edad & Estadio $^{+}$ & TS \\
\hline 1. Ansiedad &, 012 &,- 079 &, 030 \\
2. Depresión &, 005 &,- 068 &, 082 \\
3. Distrés psicológico &, 010 &,- 090 &, 064 \\
4. Miedo a la recurrencia &, 052 &, 021 &, $157^{*}$ \\
7. CDVRS &, 182 &, 053 &, $163 *$ \\
\hline $\mathrm{N}=203$ & & \\
$\begin{array}{l}\text { Prueba de Momento de Pearson } \\
{ }^{+} \text {Prueba Rho de correlación de Spearman } \\
* \text { la relación es significativa }<0,05 / * * \text { la relación es significativa }<0,01\end{array}$ \\
\hline
\end{tabular}

\section{Discusión}

El presente estudio tuvo como objetivo determinar las relaciones entre el distrés psicológico, el MRC y la CDVRS en una muestra de pacientes con cáncer de mama venezolanas. De acuerdo con los resultados encontrados, las pacientes reportaron niveles bajos distrés psicológico, que se reflejo en bajos síntomas depresivos, pero con predominio de síntomas ansiosos en niveles medios.

La prevalencia de casos de distrés psicológico elevado fue de $18 \%$. Zabora, et al. ${ }^{(10)}$ encontró que $32 \%$ de las pacientes con Cáncer de mama reportaban elevado distrés, que en comparación con lo hallado en este estudio, es bajo. La depresión elevada sólo fue reportada en un 7\%, mientras que la de ansiedad fue de $23 \%$. Los casos de ansiedad elevada reportados en este estudio entran en el rango de porcentaje de casos estimado por Greer et al. ${ }^{(41)}$ en la población general de pacientes oncológicos, el cual oscila entre $10 \%$ al $30 \%$. Tal como han señalado algunos autores ${ }^{(6,41,42)}$, la ansiedad suele ser más común en los pacientes con cáncer de mama y en pacientes 
en general, dado que está es una respuesta normal y adaptativa que se da ante eventos amenazantes, como el diagnóstico de cáncer. Los síntomas ansiosos por lo regular suelen aparecer desde el momento en que se sospecha del diagnóstico y que al mantenerse, afectan el bienestar y ajuste a lo largo de la enfermedad ${ }^{(1,42)}$.

Esto se complementa con los niveles reportados de MRC, que fueron moderados y con una prevalencia de casos elevados de $36 \%$, siendo mayor al porcentaje de ansiedad alta. Sin embargo, los casos con niveles moderados de MRC fueron de 53\%, algunos autores $^{(17-20)}$ han encontrado alrededor de $49 \%$ casos con niveles moderados. Con respecto a los niveles altos de $\mathrm{MRC}$, el porcentaje es elevado en comparación con los que se reportan en la población general, que son entre $6 \%$ y $10 \%$ de los $\operatorname{casos}^{(22)}$. Esto sugiere que la respuesta emocional ante la recurrencia de cáncer, aunque sea esperada, puede manifestarse de forma desbordante en las pacientes de la muestra de estudio. El MRC puede ser funcional en un primer momento, puesto que funge como una alerta para que los pacientes se mantengan atentos a sus controles médicos, pero cuando se vuelven disfuncionales, puede afectar su bienestar y calidad de vida ${ }^{(17)}$.

En cuanto a la CDVRS, se reportaron niveles que se encontraban en el rango normal. Las pacientes de la muestra parecen percibir menor afectación de la salud física que de la salud mental. Esto se corresponde con los datos reportados por la mayoría de ellas, en los que se evidenció que las pacientes no habían tenido dificultades físicas en los últimos tres meses y habían tenido buen manejo de los síntomas de la enfermedad, además de encontrarse activas laboralmente y desempeñando sus roles en su vida diaria. Estos niveles sugieren que la enfermedad y los tratamientos no han afectado de forma importante su bienestar ${ }^{(4,5)}$.

En tal sentido, se puede decir que la muestra de pacientes con cáncer de mama sobrevivientes reportan una CDVRS dentro del rango normal, con bajos niveles de depresión, niveles medios de ansiedad, que en suma, reportan un distrés psicológico en niveles bajos en general y unos niveles de MRC moderados. Al respecto, Contanzo et al. ${ }^{(13)}$ ha encontrado que aunque las pacientes sobrevivientes de cáncer de mama reporten un buen ajuste psicológico, pueden persistir niveles de distrés que asocian frecuentemente con preocupaciones relacionadas con la enfermedad.

Una posible explicación para la presencia de niveles elevados de MRC podría ser que las pacientes sobrevivientes de cáncer de mama, para el momento en que fueron recolectados los datos, asistían a la consulta de seguimiento médico de la enfermedad. Pues, se ha encontrado que las visitas al oncólogo y la realización de exámenes de seguimiento son dos de los activadores importante de $\mathrm{MRC}^{(19,30)}$. Ante este evento las pacientes pueden experimentar incertidumbre, que refiere a una incapacidad para determinar el significado de los acontecimientos relacionados con una enfermedad de la que no se tiene una previsibilidad de si aparecerá o no ${ }^{(43)}$, Esto sugiere que el procesamiento cognitivo o evaluación que hagan las pacientes sobre la enfermedad, especialmente los síntomas físicos que pueden llegar a manifestar en esta fase son un factor importante que ha de considerarse ${ }^{(19)}$. De acuerdo con Lee-Jones et al. ${ }^{(44)}$, el MRC varia conforme a la representación que se tiene sobre la enfermedad, en la que los estímulos activadores tanto externos (visitas al medico, exámenes diagnósticos, conversaciones sobre la enfermedad) como internos (signos y síntomas físicos y somáticos) producen la respuesta cognitiva asociada con este miedo. A raíz de la experiencia, se construye un significado sobre enfermedad que va estar influenciado por factores personales y contextuales que determinarán lo adaptativo o no de las respuestas dada por los individuos. 
Conforme a lo esperado, se encontraron asociaciones entre el distrés psicológico y el MRC, aunque con magnitud baja a excepción de la ansiedad que fue moderada, lo que da respaldo a lo hallado en estudios previos ${ }^{(23-26)}$. Para efecto de este estudio, tanto la ansiedad como el MRC se mantuvieron presentes en las pacientes sobrevivientes de cáncer de mama, lo que guarda correspondencia con lo hallado en otros estudios ${ }^{(13}$ 28) sobre la manifestación en conjunto de ambas respuestas emocionales. Esta correlación hallada parece sugerir la independencia de ambas variables, tal como lo han sugerido otros autores ${ }^{(17,20)}$. Se ha señalado que el MRC es diferente de la ansiedad, puesto que las reacciones que se manifiestan no son ante un evento irreal o irracional, sino uno realmente amenazante y del que los pacientes son conscientes ${ }^{(17)}$.

Asimismo, el distrés psicológico y MRC se asociaron con la CDVRS en las pacientes sobrevivientes de cáncer de mama. Lo que indica que al experimentar niveles elevados distrés y de MRC, la CDVRS global se deteriora, tal como se ha reportado en estudios previos $^{(18-30)}$. Algunos estudios ${ }^{(22,32)}$, reportaron que las pacientes sobrevivientes con cáncer de mama con una mejor CDVRS presentan mejor funcionamiento físico, emocional y social y una menor incidencia de MRC y distrés psicológico.

De acuerdo al perfil sociodemográfico de las pacientes, que indicó que la mayoría de ellas se encuentran en edades activas, en tiempo menor a 5 años de supervivencia, frecuentemente madres, solteras, aunque laboralmente activas, de bajo nivel socioeconómico y sin estudios superiores, son factores importantes que pueden mantener los niveles de distrés psicológico y de MRC, tal como se ha reportado en algunos estudios ${ }^{(16-30)}$. Se sabe que la interacción entre factores biológicos y psicosociales relacionados al cáncer pueden desbordar los recursos de afrontamiento de los pacientes oncológicos y dar lugar a respuestas emocionales que pueden desarrollarse en un continúo que va del distrés común hasta desórdenes de ajuste, de ansiedad o depresión ${ }^{(45)}$.

En aras de profundizar en los análisis, en este estudio se examinaron las relaciones entre el distrés psicológico y el MRC con la edad, el estadio inicial de la enfermedad y el tiempo de la supervivencia. Sólo se reportaron correlaciones positivas y estadísticamente significativas entre el MRC, la CDVRS y el tiempo de supervivencia. Lo que indica que las pacientes con cáncer de mama que tienen más años de sobrevida, tienen una percepción mayor de su CDVRS, al mismo tiempo que pueden mantener mayor preocupación por la recurrencia del cáncer, tal como se ha señalado en la literatura ${ }^{(8-30)}$. Sin embargo, considerando la fuerza de la relación, es importante que su interpretación se considere con precaución y aportarse más evidencia al respecto.

Con respecto a la relación con la edad y el estadio de la enfermedad, esto apoya lo reportado en la literatura ${ }^{(8-16,20,21,30)}$, en la que se ha reseñado que indistintamente de la edad, los niveles de distres y MRC podría manifestarse, sin embargo, se sabe que en mujeres de edades jóvenes, que son madres, reportan mayores niveles de distrés y de MRC, debido a que el cáncer suele ser más agresivo en esas edades ${ }^{(8,19-22,30)}$. El distrés psicológico puede manifestarse independientemente del estadio de la enfermedad en que se encuentren las pacientes. Sin embargo, en aquellas pacientes que se encuentran en estadios avanzados son las que han reportado mayor prevalencia de distrés psicológico ${ }^{(1-11,29)}$. En cuanto al MRC, los hallazgos son inconsistentes, pues, siguiendo la revisión de Simard et al. ${ }^{(20)}$, ellos encontraron que los indicadores pronósticos, tales como el estadio de la enfermedad, no necesariamente se encuentran asociado al MRC, registrando estudios en los que se da evidencia para tal relación como en otros en los que no. 


\section{Conclusiones}

De los resultados reportados en este estudio, se puede concluir que las pacientes reportaron niveles bajo de distrés psicológico en general, con niveles medios de $\mathrm{MRC}$ y un nivel de CDVRS dentro de los rangos normales. El distrés psicológico se encuentra asociado, aunque no en gran magnitud, con el MRC y ambos se asocian con un deterioro de la CDVRS en las pacientes sobrevivientes de cáncer de mama venezolanas. Además, se encontraron correlaciones positivas, aunque bajas, pero significativas entre el tiempo de supervivencia, la CDVRS y el MRC en esta muestra de pacientes.

La presente investigación representa un aporte al estudio de los factores emocionales y su influencia sobre la salud de los pacientes oncológicos venezolanos, especialmente los de cáncer de mama, una de las neoplasias frecuentemente diagnosticadas en el país. Es necesario seguir reportando más evidencia sobre los resultados hallados en este estudio, empleando una muestra mayor y con distintos tipos de canceres.

Algunas limitaciones de este estudio fueron el no contar con más participantes, dado que muchas pacientes que participaron no completaron todas las escalas y, por otro lado, aun cuando la escala del HADS reportó buena consistencia interna, no tenía estudios psicométricos previos en la población de pacientes oncológico, por tanto, no se pudo contar con una referencia local en cuanto a puntos de cortes que pudieran informar sobre niveles elevados o severos en distrés psicológico en la muestra de pacientes, por lo que se hace necesario contar con dichos estudios en el Venezuela.

\section{Referencias bibliográficas}

1. Urzúa A. Calidad de vida relacionada con la salud: elementos conceptuales. Rev. Med Chile 2010; 138: 358-65.

2. Rutherford C. Health - related quality of life in cancer. En: Oliver I, editor. The MASCC Textbook of cancer supportive care and survivorship. Australia: Springer; 2018. p. $109-$ 25. doi: 10.1007/978-3319-90990-5_8.

3. Die Trill M. Psicooncología. Madrid: ADES, 2003.

4. Organización Mundial de Salud - OMS. Cáncer de mama: prevención y control. En línea [Acceso el 23 de mayo de 2020]. Disponible en: http://www.who.int/topics/cancer/ breastcancer/es/.

5. Altuve AJ. Entre la prevención y la angustia. Reportaje interpretativo sobre la crisis de salud y el control del cáncer en Venezuela [Tesis de grado]. Caracas: Universidad Central de Venezuela: 2014.

6. Beatty, L. Kissane D. Anxiety and depression in women whit breast cancer. Cancer Forum 2017; 4: 55-61. doi: 10.1016/j.canep.2018.06.002

7. Bultz BD, Carlson LE. Emotional distress: the sixth vital sign in cancer care. J Clin Oncol 2005; 23:6440-1. doi: 10.1200/JCO.2005.02.3259.

8. Holland JC, Andersen B, Breitbart WS, Buchmann LO, Compas B, Deshields TL, et al. Distress management clinical practice guidelines in oncology. JNCC 2013; 11: 190-208. doi: 10.6004/jncen.2013.0027.

9. Derogatis LR, Morrow GR, Fetting J, Penman D, Piasetsky S, Schmale AM, et al. The prevalence of psychiatric disorders among cancer patiens. JAMA 1983; 249:751-7. doi: 10.4103/0973-1482.27590. 
10. Zabora J, BrintzenhofeSzoc K, Curbow B, Hooker C, Piantadosi S. The prevalence of psychological distress by cancer site. Psychooncology 2001; 10:19-28. doi: 10.1002/1099-1611(200101/02)10:1<19::AID-PON501>3.0.CO;2-6.

11. Deimiling G, Kahana, B, Bowman KF, Schaefer ML. Cancer survivorship and psychological distress in later life. Psychooncology 2002; 11: 479-94. doi: 10.1002/pon.61.

12. Syrowatka A, Motulsky A, Kurteva S, Hanley JA, Dixon WG, Meguerditchian AN, et al. Predictors of distress in female breast cancer survivors: a systematic review. Breast Cancer Rev Treat 2017; 165: 229-45. doi: 10.1007/s10549-017-4290-9.

13. Costanzo ES, Lutgendorf SK, Mattes ML, Trehan S, Robinson CB, Tewfik F, et al. Adjusting to life after treatment: distress and quality of life following treatment for breast cancer. Br J Cancer 2007; 97: 1625-31. doi: 10.1038/sj.bjc.66044091.

14. Ozakinci G, Sobota A, Humphiris G. Fear of cancer recurrence among breast cancer suvivors. Curr Breast Cancer Rep 2014; 1: 219-25. doi: 10.1007/s12609-014-0153-1.

15. Thewes B, Butow P, Bell ML, Beith J, Stuart-Harris R, Grossi M, et al. Fear of cancer recurrence in young women with a history of early-stage breast cancer: a cross-selecctional study of prevalence and association with health behaviours. Support Care Cancer 2012; 20; 2651-59. doi: 10.1007./s00520-011-1371-x.

16. Ganz PA. Psychological and social aspects of breast cancer. Breast Cancer 2008; 22: 24250.

17. Herschbach P, Dinkel A. Fear of progression. En: Goerling U, editor. Psycho Oncology Germany: Springer; 2018. p. 1-30.

18. van de Wall M, van de Poll-France L, Prins J, Gielissen M. Does fear of cancer recurrence differ between cancer types? A study from the population - based PROFILES registry. Psychooncology 2016; 25: 772-8. doi: 10.1002/pon.4002.

19. Butow PN, Fardell JE, Smith AB. Fear of cancer recurrence. En: Holland JC, Breitbart WS., Butow PN, Jacobsen PB, Loscalzo MJ., McCorkle R, editors. Psycho-Oncology (3 ${ }^{\mathrm{a}}$ ed.). New York: Oxford University Press; 2015. p. 627-629.

20. Simard S, Thewes B, Humphris G, Dixon, M., Hayden, C., Mireskandari, S., et al. Fear of cancer recurrence in adult cancer survivors: a systematic review of quantitative studies. J Cancer Surviv 2013;7: 300-22. doi:10.1007/s11764-013-0272-z.

21. Mast ME. Suvivors of breast cancer: illiness uncertainty, positive reappraisal, and emotional distress. Oncol. Nurs Forum 1998; 25: 555-62.

22. Koch L, Bertram H, Eberle A, Holleczek, B., Schmid-Höpfner, S., Waldmann, A., et al. Fear of recurrence in long-term breast cancer survivors-still an issue. Results on prevalence, determinants, and the association with quality of life and depression from the Cancer Survivorship - a multi-regional population-based study. Psychooncology 2014; 23: 547-54. doi:10.1002/pon.3452.

23. Götze H, Taubenheim S, Dietz A, Lordick F, Mehnert A. Fear of cancer recurrence across the survivorship trajectory: results from a survey of adult long-term cancer survivors. Psychooncology 2019; 28: 2033-41. doi: 10.1002/pon.5188.

24. Lane BE, Garland SN, Chalifour K, Eaton G, Lebel S, Galicia J, et al. Prevalence and factors associated with fear of recurrence in a mixted simple of young adults with cancer. J. Cancer Surviv 2019; 13: 842-52. doi: 10.1007/s11764-019-00802-9.

25. Thewes B, Bell ML, Butow P, Beith F, Boyle M, Friedlander SA, et al. Psychological morbility and stress but not social factors influence level of fear of cancer recurrence in young women with early breast cancer: results of a cross-sectional study. Psychooncology 2013; 12: 2797-806. doi: 10.1002/pon.3348. 
26. Thewes B, Kaal S, Custers JA, Manten-Horst E, Jansen R, Servaes P, et al. Prevalence and correlates of high fear of cancer recurrence in late adolescents and young adults consulting a specialist adolescent and young adult (AYA) cancer service. Support Care Cancer 2018; 26: 1479-87. doi: 10.1007/s00520-017-3975-2.

27. Reed SC, Bell JF, Miglioretti DL, Nekhlyudov L, Fairman N, Joseph JG. Fear of cancer recurrence and associations with mental health status and individual characteristics among cancer survivors: Findings from a nationally representative simple. J Psychosoc Oncol 2020; 38: 125-42. doi: 10.1080/07347332.2019.1649338.

28. Sun H, Yuan Y, Zhang J, Lui T, Wang H, Garg S, et al. Fear of cancer recurrence, anxiety and depressive symptoms in adolescent and young adult cancer patients. Neuropsychiatr Dis Treat 2019; 15: 857-65. doi: 10.2147/NDT.S202432.

29. Hall DL, Jimenez RB, Perez GK, Rabin J, Quain K, Yeh GY, et al. Fear of cancer recurrence: A model examination of physical symptoms, emotional distress and health behavior change. J. Oncol Practice 2019; 15: 787-97. doi: 10.1200/JOP.1800787.

30. Crist JV, Grunfeld EA. Factors reported to influence fear of recurrence in cancer patients: a systematic review. Psychooncology 2013; 22: 978-86. doi:10.1002/pon.3114.

31. Yang Y, Li W, Wen Y, Wang H, Sun H, Liang W, et al. Fear of cancer recurrence and adolescent and young adult cancer survivors. A systematic review of the literature Psychoncology 2019; 28: 675-86. doi: 10.1002/pon.5013.

32. Dunn L, Langford DJ, Steven MP, Berman MB, Shumay DM, Kober K, et al. Trajectories of fear of recurrence in women with breast cancer. Support Care Cancer 2015; 23: 203343. doi: 10.1007/s00520-014-2513-8.

33. Hernández R, Fernández C, Baptista L. Metodología de la investigación. Mexico: McGraw Hill; 2012

34. Zigmond A, Snaith, R. The Hospital Anxiety and Depression Scale. Acta Psychiatr Scan 1983; 67: 361-70. doi:10.1111/j.1600-0447.1983.tb09716.x.

35. Rico JL, Restrepo M, Molina M. Adaptación y validación de la escala hospitalaria de ansiedad y depresión en una muestra de pacientes con Cáncer del Instituto Nacional de Cancerología de Colombia. Avances en Medición 2005; 3: 73-86.

36. Deimling GT, Albitz M, Monnin BA, Renzhofer MA, Pappada HT, Nalepa E, et al. Personality and psychological distress among older adult, long-term cancer survivors, J Psychosoc Oncol 2017; 35: 17-31. doi: 10.1080/07347332.2016.1225145

37. Gotay, CC, Muraoka, MY. Cancer-related Worries Scale [Unpublished]. 1999.

38. Muñiz J, Elosua P, Hamblenton RK. Directrices para la traducción y adaptación de los tests: segunda edición. Psicothema 2013; 25: 151-7.

39. Ware JE, Korinski, M, Kelle SD. A 12-Item Short-Form Health Survey: construction of scales and preliminary tests of reliability and validity. Med Care 1996; 34: 220-33. doi: 10.1097/00005650-19960300-00003.

40. Hernández N, Salas A, Altuve JJ. Afrontamiento, funcionamiento familiar y calidad de vida relacionada con la salud en cuidadores venezolanos de pacientes con cáncer. Psicología y Salud 2020; 30: 161-72. doi: 10.25009/pys.v30i2.2651.

41. Greer JA, MacDonald J, Traeger L. Anxiety Disorders. En: Holland JC, Breitbart WS., Butow PN, Jacobsen PB, Loscalzo MJ., McCorkle R, editors. Psycho-Oncology ( $3^{\mathrm{a}}$ ed.). New York: Oxford University Press; 2015. p. 296 - 303.

42. Jara C, Gutiérrez Y. Psicooncologia: Aportes a la compresion y la terapeutica. Chile: Nueva Mirada Ediciones, 2016

43. Mishel MH. Reconceptualization of the uncertainty in illness theory. The Journal of Nursing Scholarship 1990;22:256-62. doi: 10.1111/j.1547-5069.1990.tb00225.x. 
44. Lee-Jones C, Humphris G, Dixon R, Hatcher MB. Fear of cancer recurrence - a literature review and proposed cognitive formulation to explain exacerbation of recurrence fears. Psychooncology 1997; 6: 95-105. 10.1002/(SICI)1099-1611(199706)6:2<95::AIDPON250>3.0.CO;2-B.

45. Li M, Hales S, Rodin G. Adjustment Disorders. En: Holland JC, Breitbart WS., Butow PN, Jacobsen PB, Loscalzo MJ., McCorkle R, editors. Psycho-Oncology ( $3^{\mathrm{a}}$ ed.). New York: Oxford University Press; 2015. p.274-280. 